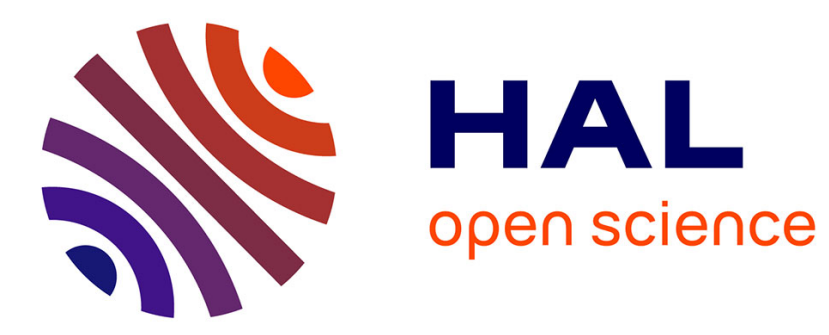

\title{
Low complexity partial selected mapping for PAPR reduction of OFDM system
}

Hyunseuk Yoo, Frédéric Guilloud, Ramesh Pyndiah

\section{To cite this version:}

Hyunseuk Yoo, Frédéric Guilloud, Ramesh Pyndiah. Low complexity partial selected mapping for PAPR reduction of OFDM system. IEEE SCVT 2010: 7th Annual Symposium on Communications and Vehicular Technology, Nov 2010, Enschede, Netherlands. hal-00565621

\section{HAL Id: hal-00565621 \\ https://hal.science/hal-00565621}

Submitted on 14 Feb 2011

HAL is a multi-disciplinary open access archive for the deposit and dissemination of scientific research documents, whether they are published or not. The documents may come from teaching and research institutions in France or abroad, or from public or private research centers.
L'archive ouverte pluridisciplinaire HAL, est destinée au dépôt et à la diffusion de documents scientifiques de niveau recherche, publiés ou non, émanant des établissements d'enseignement et de recherche français ou étrangers, des laboratoires publics ou privés. 


\title{
Low Complexity Partial Selected Mapping for PAPR Reduction of OFDM System
}

\author{
Hyunseuk Yoo, Associate Member, IEEE Frederic Guilloud, Member, IEEE, \\ and Ramesh Pyndiah, Senior Member, IEEE
}

\begin{abstract}
We present partial blind selected mapping method (P-BSLM) as a probabilistic OFDM-PAPR reduction method. The P-BSLM method generates more candidates than the classical SLM (C-SLM) method while using the same number of IFFT computations. Moreover, common stage computation in an IFFT process can reduce the computational complexity. More candidates increase the PAPR reduction capability, and give a better error performance in the presence of non-linear amplifier. This method has the maximum spectral efficiency without side information, and the phase sequence can be correctly detected using partial blind phase sequence detection.
\end{abstract}

Index Terms-OFDM, phase sequence detection, low complexity SLM, PAPR reduction.

\section{INTRODUCTION}

$\mathbf{O}$ RTHOGONAL frequency division multiplexing (OFDM) is a multi-carrier multiplexing technique, where data is transmitted through several parallel frequency subchannels at a lower rate. It has been popularly standardized in many wireless applications such as Digital Video Broadcasting (DVB), Digital Audio Broadcasting (DAB), High Performance Wireless Local Area Network (HIPERLAN), IEEE 802.11 (WiFi), and IEEE 802.16 (WiMAX).

An important drawback of OFDM based system is its high Peak-to-Average Power Ratio (PAPR) at the transmitter, requiring the use of a highly linear amplifier which leads to low power efficiency [1]. Moreover, when OFDM signal level works on the non-linear area of amplifier, the OFDM signals go through non-linear distortions and degrade the error performance.

The various approaches to alleviate this problem in OFDMbased systems can be classified into five categories: clipping effect transformation [2], [3], [4], coding [5], [6], frame superposition using reserved tones [7], expansible constellation point: tone injection [7] and active constellation extension [8], [9], and probabilistic solutions.

Probabilistic methods are distortionless without additional power increase. The principle of probabilistic methods is applicable to reduce the probability of high PAPR by generating several OFDM symbols carrying the same information and by selecting the one having the lowest PAPR. Partial transmit sequence (PTS) [10], [11], [12], selected mapping (SLM) [12], [13], [14] and interleaving [15], [16], [17] are well known probabilistic methods.

H. Yoo, F. Guilloud, and Ramesh Pyndiah are with the Department of Signal and Communications, Telecom Bretagne, Brest, France, e-mail: hyunseuk.yoo@telecom-bretagne.eu

$978-1-4244-8489-8 / 10 / \$ 26.00$ (C) 2010 IEEE
One drawback of probabilistic methods is the high computational complexity required by the number of IFFT operations: this makes it difficult the implementation for wireless handheld devices.

Another problem to be solved is the detection of the transmitted candidate. Since probabilistic methods generate multiple candidates and the best one is transmitted, if receiver does not have any information about the selected candidate, the recovery of the original data is not achievable. For the candidate recognition, there are two kinds of strategies: embedded side information [15], [16], [17] and blind detection [14]. The first solution reduces the spectral efficiency due to the embedded side information, while the second solution guarantees the maximum spectral efficiency at the expense of complicated candidate detection process (blind detection). However, this compleixty of the second solution can be solvable in a fixed base station.

In this paper, we present a low complexity partial blind selected mapping method (P-BSLM) as a probabilistic PAPR reduction method of OFDM system. P-BSLM generates more candidates than classical SLM (C-SLM) by using the same number of IFFT operations, and the numerous candidates reduce the PAPR more efficiently. In addition, we present partial blind candidate detection method.

The paper is organized as follows. In Section II, we briefly overview classical SLM (C-SLM) method for OFDM system. In Section III, we describe the P-BSLM. In Section IV, the low complexity design is discussed. In Section V, we present our numerical results, and finally we conclude this paper in Section VI.

\section{OVERVIEW OF SLM}

Selected mapping (SLM) method is a well known probabilistic technique to reduce the PAPR. In this method, the original modulated data $\overline{\mathbf{X}}=\left\{\bar{X}_{1}, \cdots, \bar{X}_{N}\right\}$ is multiplied by $U$ phase sequences $\mathbf{P}^{(u)}=\left\{e^{j \phi_{1}^{(u)}}, \cdots, e^{j \phi_{N}^{(u)}}\right\}$, to generate $U$ candidates (frequency domain) given by

$$
\mathbf{X}^{(u)}=\mathbf{P}^{(u)} \otimes \overline{\mathbf{X}},
$$

where $\otimes$ is component-wise multiplication and $\phi_{m}^{(u)} \in(0,2 \pi]$ for $u=\{1, \cdots, U\}$.

Then, by an inverse discrete Fourier transform (IDFT) operation, $U$ candidates (time domain) $\mathbf{x}^{(u)}=\left\{x_{1}^{(u)}, \cdots, x_{N}^{(u)}\right\}$ are obtained as follows:

$$
x_{m}^{(u)}=\frac{1}{\sqrt{N}} \sum_{k=1}^{N} X_{k}^{(u)} e^{j \frac{2 \pi(k-1)(m-1)}{N}},
$$




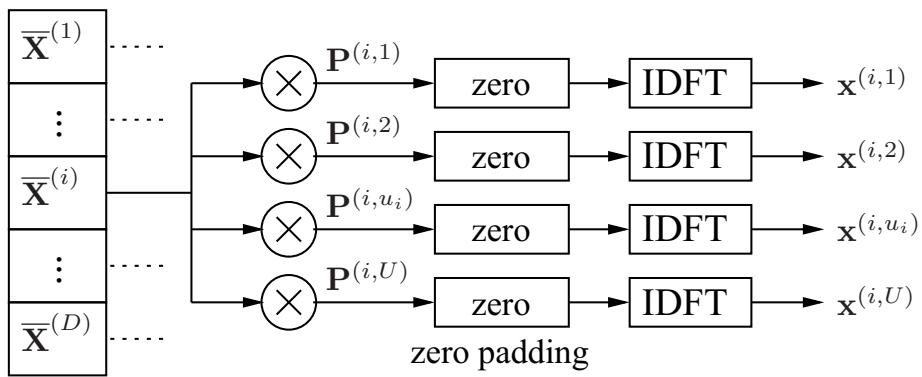

Fig. 1. IDFT computation for P-BSLM, where $u_{i} \in\{1, \cdots, U\}$, and $D=2^{\alpha}$.

and, the best $\left(u_{0}\right.$ th) candidate having the minimum PAPR is selected and transmitted, where

$$
u_{0}=\underset{u \in\{1, \cdots, U\}}{\arg \min } \frac{\max \left|\mathbf{x}^{(u)}\right|^{2}}{\mathrm{E}\left[\left|\mathbf{x}^{(u)}\right|^{2}\right]} .
$$

The computation of an IDFT can be implemented by an inverse fast fourier transform (IFFT) circuit. Then, $U$ IDFT processings are required to generate $U$ candidates.

\section{Partial Blind Selected mapping (P-BSLM) DESCRIPTION}

\section{A. Transmitter}

We divide the original modulated data $\overline{\mathbf{X}}$ into $D=2^{\alpha}$ subvectors $\overline{\mathbf{X}}=\left\{\overline{\mathbf{X}}^{(1)}, \overline{\mathbf{X}}^{(2)}, \cdots, \overline{\mathbf{X}}^{(D)}\right\}$, where each subvector $\overline{\mathbf{X}}^{(i)}=\left\{\bar{X}_{1}^{(i)}, \bar{X}_{2}^{(i)}, \cdots, \bar{X}_{2^{\beta}}^{(i)}\right\}$ has $2^{\beta}$ elements for $i \in\left\{1, \cdots, 2^{\alpha}\right\}, \alpha$ and $\beta$ being two positive integers such that $\alpha+\beta=\log _{2} N=n$.

As seen in Figure 1, each subvector $\overline{\mathbf{X}}^{(i)}$ is componentwisely multiplied by the $i$ th phase vector

$$
\mathbf{P}^{(i, u)}=\left\{e^{j \phi_{1}^{(i, u)}}, e^{j \phi_{2}^{(i, u)}}, \cdots, e^{j \phi_{2}^{(i, u)}}\right\}
$$

to generate $U$ candidates $\mathbf{X}^{(i, u)}$ for each subvector, where $\phi_{m}^{(i, u)} \in(0,2 \pi], i \in\left\{1, \cdots, 2^{\alpha}\right\}, u \in\{1, \cdots, U\}, m \in$ $\left\{1, \cdots, 2^{\beta}\right\}$. Note that $\mathbf{X}^{(i, u)}$ is a vector with $2^{\beta}$ elements.

The $U$ candidates $\mathbf{X}^{(i, u)}$ are zero-padded and an N-point IDFT is performed, as follows:

$$
\mathbf{x}^{(i, u)}=\operatorname{IDFT}_{N}\left(\left\{\mathbf{Z}^{(1)}, \cdots, \mathbf{X}^{(i, u)}, \cdots, \mathbf{Z}^{(D)}\right\}\right)
$$

to generate $U$ partial candidates in the time domain, where $\mathbf{Z}^{(i)}$ is an all zero vector of length $2^{\beta}$ placed on the $i$ th division (corresponding to $\overline{\mathbf{X}}^{(i)}$ ). Notice that $\mathbf{x}^{(i, u)}$ is a vector with $N=2^{\beta} \cdot 2^{\alpha}$ elements.

Then, the time domain candidates are given by

$$
\mathcal{C}\left(u_{1}, \cdots, u_{D}\right)=\mathbf{x}^{\left(1, u_{1}\right)}+\mathbf{x}^{\left(2, u_{2}\right)}+\cdots+\mathbf{x}^{\left(D, u_{D}\right)},
$$

for $u_{1}, u_{2}, \cdots, u_{D} \in\{1,2, \cdots, U\}$, where there exist $U^{D}$ possible combinations. The combination which has the lowest PAPR is selected:

$$
\left\{u_{1}^{\prime}, \cdots, u_{D}^{\prime}\right\}=\underset{\left\{u_{1}, \cdots, u_{D}\right\}}{\arg \min }\left(\operatorname{PAPR}\left\{\mathcal{C}\left(u_{1}, \cdots, u_{D}\right)\right\}\right),
$$

and transmitted:

$$
\mathbf{x}^{\prime}=\mathcal{C}\left(u_{1}^{\prime}, \cdots, u_{D}^{\prime}\right) .
$$

\section{B. Receiver: P-BSLM phase sequence detection}

The received vector, $\mathbf{r}=\mathbf{x}^{\prime}+\mathbf{n}$, is DFTed, where $\mathbf{n}$ is the noise term. Then, we have $\widetilde{\mathbf{X}} \triangleq \operatorname{DFT}_{N}(\mathbf{r})$,

$$
\begin{aligned}
\widetilde{\mathbf{X}}= & \left\{\mathbf{X}^{\left(1, u_{1}^{\prime}\right)}, \mathbf{X}^{\left(2, u_{2}^{\prime}\right)}, \cdots, \mathbf{X}^{\left(D, u_{D}^{\prime}\right)}\right\} \\
& +\operatorname{DFT}_{N}(\mathbf{n}) \\
= & \left\{\widetilde{\mathbf{X}}^{\left(1, u_{1}^{\prime}\right)}, \widetilde{\mathbf{X}}^{\left(2, u_{2}^{\prime}\right)}, \cdots, \widetilde{\mathbf{X}}^{\left(D, u_{D}^{\prime}\right)}\right\} .
\end{aligned}
$$

The received vectors should be multiplied by the conjugate phase sequences $\mathbf{P}^{*(i, u)}$ on the $D$ subvectors for the recovery of original data, as follows:

$$
\widehat{\mathbf{X}}^{(i)}=\mathbf{P}^{*\left(i, u_{i}^{\prime}\right)} \otimes \mathbf{X}^{\left(i, u_{i}^{\prime}\right)}+\mathbf{N}^{(i)}
$$

for $i \in\{1, \cdots, D\}$, where $\mathbf{N}^{(i)}$ is a noise term which falls on the $i$ th frequency division.

To recover the original data, the phase sequences at the transmitter should be correctly detected at the receiver. Without side information, the phase sequence can be detected by the blind detection algorithm (BSLM) [14]. Especially, in this case, there are $D$ divisions on an OFDM symbol vector, and each division is multiplied by an individual phase sequence. Therefore, we should detect $D$ partial SLM phase sequences, as follows:

${\widehat{u^{\prime}}}_{i}=$

$$
\underset{u \in\{1, \cdots, U\}}{\arg \min }\left[\sum_{m=1}^{2^{\beta}} \min _{\mathfrak{G} \in Q_{Q A M}}\left|\widetilde{X}_{m}^{(i, u)} \otimes P_{m}^{*}(i, u)-\mathfrak{G}\right|^{2}\right]
$$

for $i \in\{1, \cdots, D\}$, where $\mathfrak{G}$ represents a QAM symbol.

\section{LOW COMPLEXITY DESIGN}

\section{A. Adaptability for low complexity SLM structure}

As seen in Section III, P-BSLM requires $U 2^{\alpha}=U D$ IFFT computations for generating $U^{D}$ time domain combinations, while the conventional SLM requires $U^{D}$ IFFT computations for generating $U^{D}$ time domain candidates [13], [14]. Then, with a small number of IFFT computations, we can generate numerous time domain candidates, and thus reduce the PAPR.

Furthermore, P-BSLM is adaptable to low complexity structures by using the successive doubling method presented in [18]. For example, in the case that $i=1$ and $D=4$ (see 
Figure 1), the IFFT circuit input is given by

$$
\begin{aligned}
& \left\{\mathbf{P}^{\left(1, u_{1}\right)} \otimes \overline{\mathbf{X}}^{(1)}, \mathbf{Z}^{(2)}, \mathbf{Z}^{(3)}, \mathbf{Z}^{(4)}\right\} \\
= & \left\{\mathbf{P}^{\left(1, u_{1}\right)}, \mathbf{P}^{\left(1, u_{1}\right)}, \mathbf{P}^{\left(1, u_{1}\right)}, \mathbf{P}^{\left(1, u_{1}\right)}\right\} \\
\otimes & \left\{\overline{\mathbf{X}}^{(1)}, \mathbf{Z}^{(2)}, \mathbf{Z}^{(3)}, \mathbf{Z}^{(4)}\right\}
\end{aligned}
$$

for $u_{1} \in\{1, \cdots, U\}$.

In [18], an $N$-point IFFT computation contains $n=$ $\log _{2} N=\alpha+\beta$ stages, where $\alpha$ stages are used for the common part and $\beta$ stages are used for generating multiple candidates, as seen in Figure 2. In this method, the common part ( $\alpha$ stage) is processed only once, and $U$ candidates are generated by processing $U$ times the remaining parts ( $\beta$ stages), reducing the computational complexity. According to the successive doubling method on decimation in frequency algorithm, the phase sequence has a form, such as $\left\{\mathbf{P}^{(i, u)}, \mathbf{P}^{(i, u)}, \cdots, \mathbf{P}^{(i, u)}, \mathbf{P}^{(i, u)}\right\}, u \in\{1, \cdots, U\}$, which has $2^{\alpha}$ same subvectors, and each subvector has $2^{\beta}$ phase elements. This format is exactly the same as $\left\{\mathbf{P}^{\left(1, u_{1}\right)}, \mathbf{P}^{\left(1, u_{1}\right)}, \mathbf{P}^{\left(1, u_{1}\right)}, \mathbf{P}^{\left(1, u_{1}\right)}\right\}$ in (12), when $\alpha=2$ (two common stages are considered).

For the general case, the phase sequences are given by

$$
\left\{\mathbf{P}^{(i, u)}, \mathbf{P}^{(i, u)}, \cdots, \mathbf{P}^{(i, u)}\right\},
$$

for $i \in\left\{1, \cdots, D=2^{\alpha}\right\}, u \in\{1, \cdots, U\}$, which has the suitable form for the successive doubling method [18].

In [18], the phase sequences are composed of $\mathbf{P} \in$ $\{+1,-1\}$, as a row of Hadamard matrix. However, in that case, the phase sequence detection is impossible without side information. Accordingly, it should be a random phase sequence $\mathbf{P}^{(i, u)} \in\left\{e^{j \phi_{m}^{(i, u)}}\right\}$, where $\phi_{m}^{(i, u)} \in(0,2 \pi], i \in$ $\{1, \cdots, D\}, u \in\{1, \cdots, U\}, m \in\left\{1, \cdots, 2^{\beta}\right\}$.
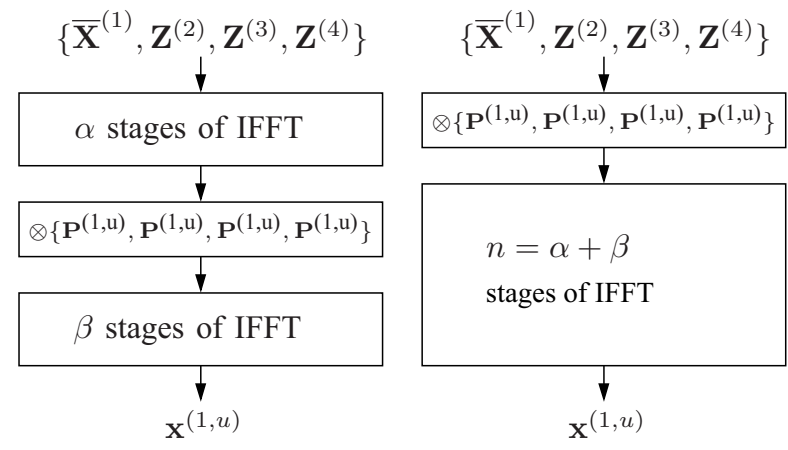

(a) Low complexity IFFT structure (b) Equivalent IFFT circuit (classical on DIF algorithm SLM)

Fig. 2. Equivalent IFFT circuit on decimation in frequency algorithm, where the phase sequence should have a form $\left\{\mathbf{P}^{(i, u)}, \mathbf{P}^{(i, u)}, \cdots, \mathbf{P}^{(i, u)}\right\}$

\section{B. Computational complexity}

To compare the computational complexity, there are two points of view: the computational complexity for the same number of candidates, and the number of candidates for the same computational complexity.

For the first point of view, we take into consideration $U^{D}=U^{2^{\alpha}}$ candidates. The classical SLM [13] requires
$U^{D}$ IFFT computations to generate $U^{D}$ candidates, while the P-BSLM needs only UD IFFT computations. Furthermore, when we use the phase sequences as (13) in a form of the succeissive doubling method [18], the P-BSLM needs $U D \cdot\left[1-\frac{\alpha}{n}\left(1-\frac{1}{U}\right)\right]$ IFFT computations, where an IFFT computation is assumed to have $2^{n-1} n$ complex multiplications and $2^{n} n$ complex additions. Then, the computational complexity reduction ratio (CCRR) is defined by [18]

$$
\begin{aligned}
\mathrm{CCRR} & \triangleq\left(1-\frac{\mathcal{C}_{\mathrm{P}-\mathrm{BSLM}}}{\mathcal{C}_{\mathrm{SLM}}}\right) \times 100(\%) \\
& =\left(1-\frac{U D \cdot\left[1-\frac{\alpha}{n}\left(1-\frac{1}{U}\right)\right]}{U^{D}}\right) \times 100,
\end{aligned}
$$

where $\mathcal{C}_{\mathrm{P}-\mathrm{BSLM}}$ is the computational complexity of P-BSLM, and $\mathcal{C}_{\text {SLM }}$ is the computational complexity of classical SLM [13]. Table I shows the CCRR (\%) values of P-BSLM. In the table, we can see that, when $U$ increases, the CCRR values increase largely, and especially, when $\alpha$ increases, the CCRR values sharply approach the value of $100 \%$.

For the second point of view, it is assumed that the system is capable to calculate $U \cdot D$ IFFT operations for the PAPR reduction. Then, the conventional SLM can generate $U \cdot 2^{\alpha}$ candidates, while P-BSLM can generate $U^{D}$ candidates (combinations), resulting in the gain of the number of candidates, $U^{D}-U D$.

\section{NUMERICAL RESULTS}

Figure 3 shows the complementary cumulative distribution function (CCDF) representing the probability that the PAPR value $\lambda$ is higher than a given threshold PAPR value $\bar{\lambda}$, $\operatorname{Prob}\{\lambda>\bar{\lambda}\}$, where $\lambda \triangleq \frac{\max \left[\left|\mathbf{x}^{\prime}\right|^{2}\right]}{\mathrm{E}\left[\left|\mathbf{x}^{\prime}\right|^{2}\right]}$. In the figure, the dashed lines represent $N=256$-point IFFT, and the solid lines represent $N=1024$-point IFFT. For the simulation, we used the P-BSLM which has the parameters $(\alpha=2, U=2)$ with 16 candidates, and C-SLM (classical SLM) method which has 8 candidates. For C-SLM, 8.0 IFFTs computation is required, and for P-BSLM, 7.0 and 7.2 IFFTs computations are required for $N=256$ and $N=1024$, respectively, due to the "low complexity adaptable structure" as mentioned in Section IV.

Despite the fact that P-BSLM has lower complexity than the compared C-SLM, P-BSLM (o) gives better CCDF performance than C-SLM $(\square)$ as seen in the figure.

Figure 4 shows Blind Detection Error Rate (BDER), which is the probability that the detected phase sequence at the receiver does not match the phase sequence applied at the transmitter. For the simulation, P-BSLM with the parameters $(\alpha=2, U=2)$ is considered for $N=256$ and $N=1024$. In this case, there exist $2^{\alpha}=4$ subvectors, and each subvector is (component-wise) multiplied by $U=2$ sequences. For the detection of each phase sequence, $2^{\beta}$ subcarriers are taken into consideration by the detection criteria (11), where $2^{\beta}=64$ for $N=256$, and $2^{\beta}=256$ for $N=1024$. In the figure, the solid lines $(N=1024)$ show much better performance than the dashed lines $(N=256)$. Because, while the phase sequences are detected by referring to only 64 subcarriers for $N=256$, 256 subcarriers are referred for the phase detection in the case 
TABLE I

COMPUTATIONAL COMPLEXITY REDUCTION RATIO FOR GENERATING $U^{2^{\alpha}}$ CANDIDATES (COMBINATIONS).

\begin{tabular}{c||c|c|c|c|c|c|c|c|c|c|c|c}
\hline \hline \multirow{2}{*}{$\alpha$} & \multicolumn{3}{c|}{$N=1024(n=10)$} & \multicolumn{3}{c|}{$N=2048(n=11)$} & \multicolumn{3}{c|}{$N=4096(n=12)$} & \multicolumn{3}{c}{$N=8192(n=13)$} \\
\cline { 2 - 14 } & $\mathrm{U}=2$ & $\mathrm{U}=4$ & $\mathrm{U}=8$ & $\mathrm{U}=2$ & $\mathrm{U}=4$ & $\mathrm{U}=8$ & $\mathrm{U}=2$ & $\mathrm{U}=4$ & $\mathrm{U}=8$ & $\mathrm{U}=2$ & $\mathrm{U}=4$ & $\mathrm{U}=8$ \\
\hline$\alpha=1$ & 5.0 & 53.8 & 77.2 & 4.5 & 53.4 & 77.0 & 4.2 & 53.1 & 76.8 & 3.8 & 52.9 & 76.7 \\
\hline$\alpha=2$ & 55.0 & 94.7 & 99.4 & 54.5 & 94.6 & 99.3 & 54.2 & 94.5 & 99.3 & 53.8 & 94.5 & 99.3 \\
\hline$\alpha=3$ & 94.7 & 100.0 & 100.0 & 94.6 & 100.0 & 100.0 & 94.5 & 100.0 & 100.0 & 94.5 & 100.0 & 100.0 \\
\hline$\alpha \geqq 4$ & 100.0 & 100.0 & 100.0 & 100.0 & 100.0 & 100.0 & 100.0 & 100.0 & 100.0 & 100.0 & 100.0 & 100.0 \\
\hline \hline
\end{tabular}

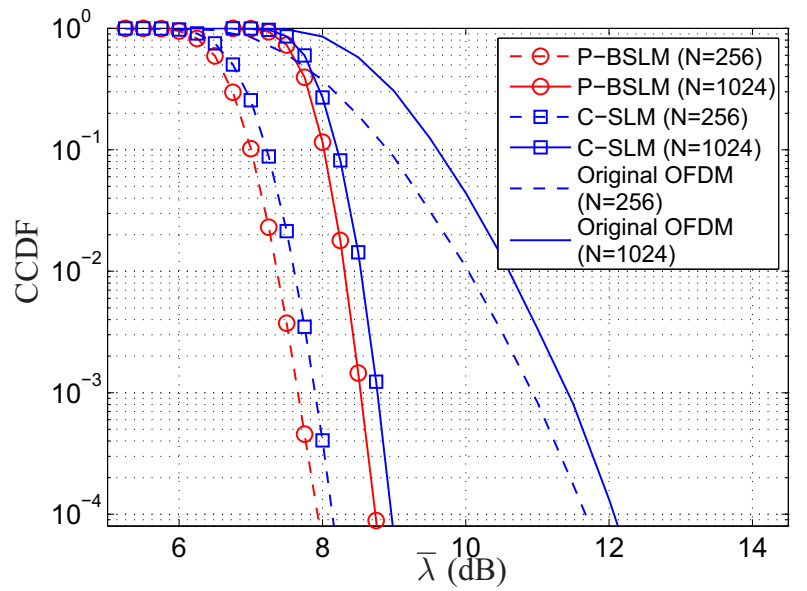

Fig. 3. Complementary Cumulative Distribution Function (CCDF) performance comparison. For the simulation, P-BSLM has the parameters $(\alpha=2$, $U=2$ ) which necessitate $U \cdot 2^{\alpha}=8$ IFFT computations. For the comparison, classical SLM (C-SLM) method with 8 IFFT computations is used. In both cases, QPSK modulation is used.

$N=1024$. In other words, when $N$ is large, the refered subcarriers for the phase sequence detection become numerous, rendering more accurate phase sequence detection. In addition, the figure compares the BDER of P-BSLM receiver with nonlinear amplifier (soft-limiter), where the transmitted signal is clipped at $2 \mathrm{~dB}$ and $5 \mathrm{~dB}$ of PAPR threshold [17].

Figure 5 and Figure 6 represent the Bit Error Rate (BER) performance over an AWGN channel and a Rayleigh fading channel, respectively. In the figures, the original OFDM performance is compared with two SLM models: Classical SLM (C-SLM) using 8 IFFTs for 8 candidates, and P-BSLM using $U 2^{\alpha}=8$ IFFTs for $U^{2^{\alpha}}=16$ candidates, where P-BSLM has the parameters $(\alpha=2, U=2)$. For a fair comparison of both SLM models, the blind phase sequence detection method is used without side information. For the simulation, the frequency domain signal is modulated by 64-QAM symbols, and 1024 subcarriers $(N=1024)$ are considered. The OFDM symbol is clipped at $\bar{\lambda}=5.5 \mathrm{~dB}$ by a soft-limiter [17], and this clipping causes the BER error floor in the figure.

When we use the SLM, PAPR value is reduced, and then, the clipping distortion is reduced, resulting in a lower error floor. For the two SLM methods (C-SLM and P-BSLM), using the same number of IFFT, P-BSLM shows better CCDF performance than C-SLM as seen in Figure 3. Consequently, P-BSLM shows better BER performance than C-SLM as in Figure 5 and Figure 6 over both the AWGN channel and the

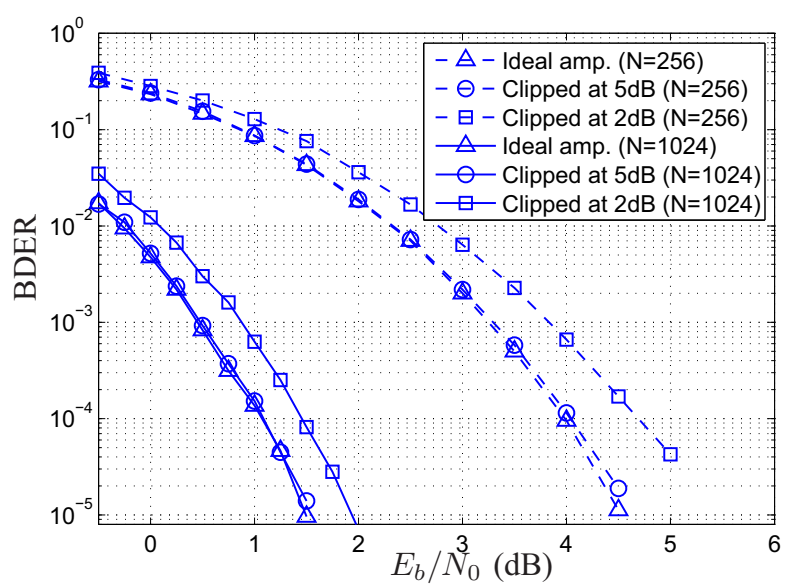

Fig. 4. Blind Detection Error Rate (BDER) for phase sequence over AWGN channel. For the simulation, P-BSLM with the parameters $(\alpha=2, U=2)$ is considered, when $N=256$ (dashed line) and $N=1024$ (solid line). For the amplifier model, soft-limiter model is considered, and the transmitted signal is clipped at $2 \mathrm{~dB}$, and $5 \mathrm{~dB}$.

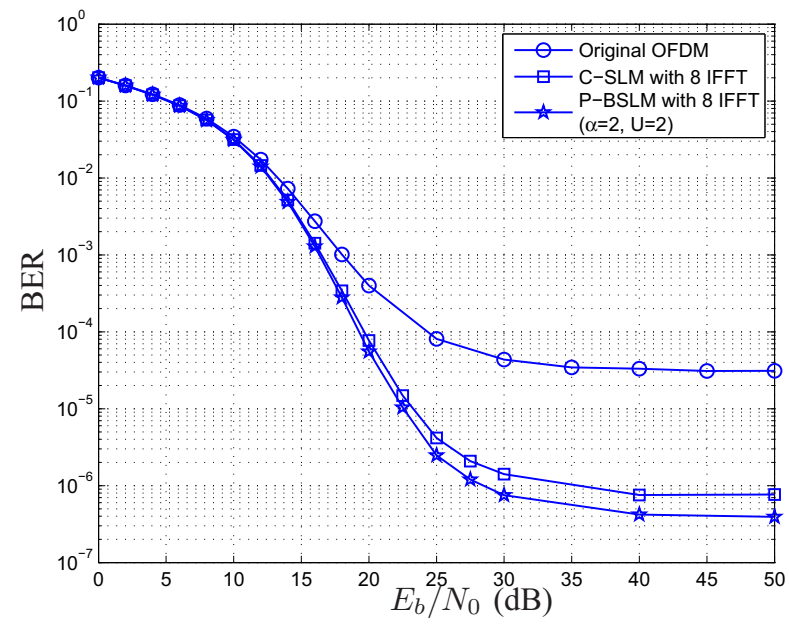

Fig. 5. Bit Error Rate (BER) comparison over AWGN channel. For the simulation, classical SLM (C-SLM) using 8 IFFTs and P-BSLM using 8 IFFTs with the parameters ( $\alpha=2, U=2)$ are compared, where $N=1024$ is considered, and the signal is modulated by 64-QAM. The transmitted signal is clipped at $5.5 \mathrm{~dB}$ by the soft-limiter.

Rayleigh fading channel. 


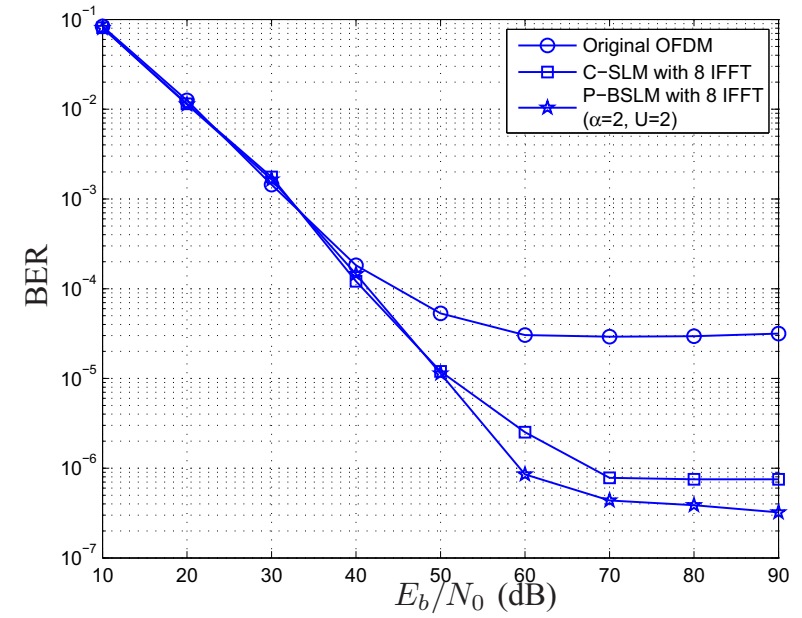

Fig. 6. BER comparison in a Rayleigh fading channel, where the same simulation parameters as in Figure 5 are considered, and perfect channel estimation (CSI) is assumed.

\section{CONCLUSION}

We present Partial Blind Selected Mapping (P-BSLM) method as a probabilistic PAPR reduction method. Conventional SLM generates $U^{D}$ candidate using $U^{D}$ IFFT computations. However, our P-BSLM generates $U^{D}$ candidates using $U D$ IFFT computations, and it reduces the PAPR when the same number of IFFT operations is considered.

The P-BSLM has an adaptive form for low complexity scheme using the successive doubling method in [18], and which makes it possible to lower the computational complexity.

The P-BSLM has the maximum spectral efficiency due to no side information. In spite of no side information, using the blind phase sequence detection method, the phase sequence can be correctly detected when a large number of subcarriers are considered.

Simulation results show that, when the same number of IFFT is used, P-BSLM achieves better CCDF performance than classical SLM (C-SLM). In the presence of a non-linear amplifier, P-BSLM thus shows better BER performance over both the AWGN channel and the Rayleigh fading channel.

\section{REFERENCES}

[1] S. H. Han and J. H. Lee, "An overview of peak-to-average power ratio reduction techniques for multicarrier transmission," IEEE Wireless Communications, pp. 56-65, April 2005.

[2] R. O'Neill and L. B. Lopes, "Envelope variations and spectral splatter in clipped multicarrier signals," in IEEE PIMRC '95, Toronto, Canada, 1995.

[3] D. Kim and G. L. Stuber, "Clipping noise mitigation for OFDM by decision-aided reconstruction," IEEE Comm. Lett., vol. 3, pp. 4-6, Jan. 1999.

[4] H. Saeedi, M. Sharif, and F. Marvasti, "Clipping noise cancellation in OFDM systems using oversampled signal reconstruction," IEEE Comm. Lett., vol. 6, pp. 73-75, Feb. 2002.

[5] A. E. Jones, T. A. Wilkinson, and S. K. Barton, "Block coding scheme for reduction of peak to mean envelope power ratio of multicarrier transmission scheme," Electron. Lett., vol. 30, pp. 2098-2099, Dec. 1994.
[6] A. E. Jones and T. A. Wilkinson, "Combined coding for error control and increased robustness to system nonlinearities in OFDM," in IEEE VTC '96, Atlanta, GA, 1996.

[7] J. Tellado and J. Cioffi, "Peak power reduction for multicarrier transmission," in IEEE CTMC, GLOBECOM '98, Sydney, Australia, 1998.

[8] D. L. Jones, "Peak power reduction in OFDM and DMT via active channel modification," in Asilomar Conference on Signals, Systems, and Computers, 1999.

[9] B. S. Krongold and D. L. Jones, "Par reduction in OFDM via active constellation extension," IEEE Trans. Broadcast., vol. 49, pp. 258-268, Sept. 2003.

[10] S. H. Muller and J. B. Huber, "OFDM with reduction peak to average power ratio by optimum combination of partial transmit sequences," Electron. lett., vol. 33, pp. 368-369, Feb. 1997.

[11] Y. Xiao, X. Lei, Q. Wen, and S. Li, "A class of low complexity PTS techniques for PAPR reduction in OFDM systems," IEEE Signal Processing Lett., vol. 14, no. 10, pp. 680 -683, Oct. 2007.

[12] S. H. Muller and J. B. Huber, "A comparison of peak power reduction schemes for OFDM," in GLOBECOM, 1997.

[13] R. W. Bauml, R. Fischer, and J. B. Huber, "Reducing the peak to average power ratio of multicarrier modulation by selected mapping," Electron. lett., vol. 32, pp. 2056-2057, Oct. 1996.

[14] A. D. S. Jayalath and C. Tellambura, "A blind slm receiver for PARreduced OFDM," in VTC 2002, Sept. 2002.

[15] — , "Reducing the peak-to-average power ratio of orthogonal frequency division multiplexing signal through bit or symbol interleaving," Electron. Lett., vol. 36, pp. 1161-1163, June 2000.

[16] _ "Peak-to-average power ratio reduction of an OFDM signal using data permutation with embedded side information," in ISCAS 2001, 2001.

[17] H. Ochiai and H. Imai, "Performance of the deliberate clipping with adaptive symbol selection for strictly band-limited OFDM systems," IEEE Journal on selected areas in communications, vol. 18, pp. 2270 2277, Nov. 2000.

[18] D.-W. Lim, J.-S. No, C.-W. Lim, and H. Chung, "A new SLM OFDM scheme with low complexity for PAPR reduction," IEEE Signal Processing Lett., vol. 12, no. 2, pp. 93 -96, Feb. 2005. 